Running head: Misleading gesture in children's eyewitness interviews

Handmade Memories: The Robustness of the Gestural Misinformation Effect in Children's Eyewitness Interviews.

\author{
Elizabeth Kirk $^{\mathrm{a}^{*},}$ Daniel Gurney ${ }^{\mathrm{b},}$ Rebecca Edwards ${ }^{\mathrm{b},}$ Chris Dodimead ${ }^{\mathrm{b}}$

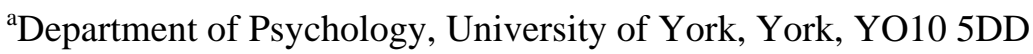

${ }^{b}$ Department of Psychology, University of Hertfordshire, College Lane, Hatfield, Hertfordshire, AL10 9AB, UK.

*Corresponding author

email: elizabeth.kirk@york.ac.uk, mobile: +(44)7961 735064 
Running head: Misleading gesture in children's eyewitness interviews

\begin{abstract}
An interviewer's gestures can convey misleading information and subsequently cause inaccuracies in the reporting of an event by both adults and children. We investigated the robustness of the gestural misinformation effect, examining the extent to which an interviewer's gestures mislead children under conditions that would normally buffer them against verbal suggestibility (strength of memory trace, age, and verbal ability). Children (a younger sample aged 2-4 years, $n=30$; and an older sample aged 7-9 years, $n=26$ ) were exposed to a videotaped event and questioned immediately, having been allocated randomly to either an accurate gesture condition (gestures consistent with observed events, e.g. "What was the lady wearing?” plus a 'hat' gesture) or a misleading gesture condition (“What was the lady wearing?” plus a 'gloves’ gesture). Children were susceptible to the gestural misinformation effect even when questioned immediately after witnessing the event, regardless of age and verbal ability. These findings reveal new insights into the robustness of the gestural misinformation effect in children's eyewitness interviews.
\end{abstract}

Keywords: eyewitness testimony, suggestibility, gesture, nonverbal, false memory 


\section{Handmade Memories: The Robustness of the Gestural Misinformation Effect in Children's Eyewitness Interviews}

It has been well established that exposure to leading verbal suggestions can influence the accuracy of eyewitness reports (Loftus \& Hoffman, 1989; see Loftus, 2005, for a review). While the vast majority of research in this area focuses on verbal information as a source of misinformation, research more recently has revealed the pervasive power of nonverbal behavior to mislead. The gestures of an interviewer can corrupt an individual's eyewitness report leading to inaccuracies and even falsities in the witnesses' long-term recollections of events. This gestural misinformation effect has been observed in both adults (Gurney, Pine, \& Wiseman, 2013) and children (Broaders \& Goldin-Meadow, 2010).

The suggestibility of children and the interviewing conditions that degrade the accuracy of their reports has been investigated heavily (See Bruck \& Melnyk, 2004; Ceci \& Bruck, 1993; 1999; Clarke-Stewart, Malloy, \& Allhusen, 2004, for reviews). However, it is only recently that the role of hand gestures during investigative interviews and the extent to which these gestures could mislead children has been investigated. Broaders and Goldin-Meadow (2010) tested the extent to which semantic information conveyed by an interviewer's gesture could influence children's eyewitness reports. Children, aged 5 to 6 years, were visited in their classroom by a musician and were interviewed repeatedly about this novel event over a 10-12 week period, with the first of five interviews conducted two weeks after the visit. Each interview comprised questions that were accompanied by misleading gestures (i.e., gestures that conveyed non-occurring information), for example "What else was he wearing?” plus a 'put on hat' gesture. Children were misled by the gestures and provided affirming responses about non-occurring events. The misleading effect of gesture was found to be as strong as suggestive verbal questioning. Children gave just as many incorrect (non-occurring) responses when asked open-ended questions accompanied by a misleading gesture as when asked specific questions (“Was he wearing a hat?”) in speech only. This research provides good evidence to demonstrate that the veracity and validity of a child's eyewitness account is vulnerable to nonverbal influence.

A great deal is understood about the factors that buffer children against the effects of verbal influence (see Bruck \& Melnyk, 2004; Klemfuss \& Ceci, 2012, for reviews). However, as yet we do not know whether these same factors protect children from the misleading effects of gesture. In the present study we extend the 
Running head: Misleading gesture in children's eyewitness interviews

work of Broaders and Goldin-Meadow (2010) to explore whether factors related to a child's vulnerability to verbal influence also predict susceptibility to nonverbal influence. We propose three factors that may affect the extent to which children are misled by gesture: strength of memory trace, age, and verbal ability.

According to the memory trace strength theory (Pezdek \& Roe, 1995), stronger memories are more resistant to suggestibility than weaker memories. Children with weaker memories of an original event have been shown to be more susceptible to verbal suggestions (King \& Yuille, 1987; Warren, Hulse-Trotter, \& Tubbs, 1991) and this is also true of adults (Kassin, Ellsworth, \& Smith, 1989; Penrod, Loftus, \& Winkler, 1982). This literature provides a convincing case that a weak memory trace leads to greater susceptibility to verbal influence. Could the memory trace theory account for the robust effects of nonverbal influence reported by Broaders and Goldin-Meadow (2010)? Because children in this study were interviewed between two weeks and three months post-event, it is possible that the children's memory traces had decayed over time, and were thus weaker and more vulnerable to intrusion. Alternatively, the gestural misinformation effect may be robust to the extent that children will be misled by gesture even when their memory trace is strong. The latter is plausible given the differences in how information from speech and gesture is processed; while misleading information from speech can often be identified, gestures are not often attended to explicitly, yet meaning can still be gleaned from them (Gullberg \& Holmqvist, 2002; Gullberg \& Kita, 2009). We questioned whether children would be misled by gesture when questioned once, immediately after the event, when their memory trace is presumed strong. Furthermore, we asked children to recall what they could remember immediately after viewing the event and tested whether there was a relationship between the amount of accurate information that they reported and the extent to which they were misled subsequently by gesture during questioning. Previous research has found that the fewer details of the event that children remember, the more suggestible they are (Marche, 1999; Marche \& Howe, 1995; McFarlane, Powell, \& Dudgeon, 2002; Pezdek \& Roe, 1995). Thus, if gesture exerts its effects in the same way as speech then we would expect a negative relation between the amount of remembered information and suggestibility to nonverbal influence.

Additionally, we questioned whether there are age-related differences in children's susceptibility to being misled by gesture. Younger children are significantly more susceptible to a variety of suggestive or misleading verbal influences than older children (Alexander et al., 2002; Burgwyn-Bailes, Baker-Ward, 
Running head: Misleading gesture in children's eyewitness interviews

Gordon, \& Ornstein, 2001; Ceci \& Bruck, 1993; Geddie, Fradin, \& Beer, 2000, Roberts \& Blades, 1999). If these trends present for nonverbal suggestion, this would suggest the two modalities exert influence in similar ways. Alternatively, if there are no age differences in children's susceptibility to being misled by gesture, then we can argue that gesture is more pervasive than verbal influence. We sought to explore age-related differences in children's vulnerability to nonverbal suggestion by testing children both younger and older than the Broaders and Goldin-Meadow sample (aged five to six), allowing us to identify any developmental differences in the processing of misleading gestures by children aged between three and nine.

The heightened vulnerability of younger children to verbal suggestion has been attributed, in part, to their weaker language skills (Clarke-Stewart, Malloy, \& Allhusen, 2004; Geddie et al., 2000; McFarlane et al., 2002; Young et al., 2003). In a study of 70 five year olds, Clarke-Stewart et al. (2004) considered the contribution of a number of child and parent variables to young children's suggestibility to misleading questions. Children's verbal ability emerged as the strongest predictor of suggestibility, with a significant negative correlation between suggestibility and children's scores on the Adaptive Language Inventory (ALI), a caregiver completed measure of children’s general verbal ability (Feagans \& Farrans, 1997). Similarly, Kulkofsky and Klemfuss (2008) found 3 to 4 year old children were more likely to assent to false information when questioned if they scored lower on the ALI. It is important to note that in this study the relationship between language ability and suggestibility was independent of age. We sought to test whether verbal ability would buffer children against the misleading effects of gesture during questioning in the same way that it does for verbal suggestibility. We expected that children with weaker verbal abilities would be especially vulnerable to the gestural misinformation effect. Previous research has found that children with weaker verbal language abilities rely more on the nonverbal channel as a source of information (Kirk, Pine, \& Ryder, 2011). Thus, we predicted that children with weaker verbal skills would be more dependent on nonverbal channels and thus would glean more information (regardless of the accuracy of this information) from gesture and would incorporate this into their narratives of the event.

The present study. To summarize, we aimed to test the robustness of the gestural misinformation effect, considering the impact of misleading gesture: (1) when the memory trace is expected to be strong (by questioning children immediately after the observed event); (2) in two novel age groups, a younger sample of 
Running head: Misleading gesture in children's eyewitness interviews

children aged 3-4 and an older sample of children aged 7-8; (3) and exploring the relationship between verbal ability and susceptibility to nonverbal influence. Given the novelty of this research, our investigation of the factors that protect against verbal influence is exploratory. In the present study, all children were exposed to one videotaped stimulus. Immediately after viewing this, children were asked to recall as much as they could to provide a measure of their original event memory. An interviewer then questioned the children under one of two conditions; accurate gesture or misleading gesture.

We wanted to rule out the possibility that the gestural misinformation effect represents a demand characteristic (i.e., children concur indiscriminately with any information conveyed by an adult's gestures, under the presumption that the adult is signaling the correct answer to the child). Eyewitnesses often comply with suggestions from interviewers that are perceived to be authoritative or have greater expertise (Skagerberg \& Wright, 2009; Smith \& Ellsworth, 1987). We were interested to understand whether children would indiscriminately assent to suggestions conveyed by an adult's gesture. Therefore, an accurate gesture condition was included to allow us to compare the amount of information that children glean from gestures that confirm or contradict their event memory. If no difference is found in the amount of information that children glean from accurate and misleading gestures, this would indicate that children attend to gesture indiscriminately, supporting the argument for the gestural misinformation effect as a demand characteristic. We expected children to incorporate more information from the accurate than the misleading gestures as these are likely to trigger the child's memory for details about the event that they have encoded. While less information was anticipated to be gleaned from the misleading gesture, we expected children to be limited in their resilience to the gestural misinformation effect. Thus, including both conditions allowed us to quantify the effect of misleading gesture over and above the effect of gesture.

Of further interest is how children report misinformation presented to them and the insights this provides into how nonverbal information has been processed. Broaders and Goldin-Meadow (2010) reported that children frequently replicated the interviewer's misleading gestures during questioning. In the present study, we examined whether children's gesture production predicted the extent to which they incorporated misleading gestured information into their narratives of the original event, hypothesizing that the more children 
Running head: Misleading gesture in children's eyewitness interviews

replicated the interviewer's misleading gestures, the more likely they would be to report this false information in their subsequent verbal recall of the event.

\section{Method}

\section{Participants}

A total of 56 children participated, of which 30 children were aged between 2 and 4 years (younger sample) and 26 children were aged between 6 and 9 (older sample). Children were recruited through local nurseries, children's centers and schools in the Hertfordshire and Greater London area. The sample was ethnically diverse. The mean age of the younger sample (19 male, 11 female) was $3.50(S D=0.57$ ) years (range 32-52 months). Half of the younger children were in the accurate gesture condition $(n=15)$ and half in the misleading gesture condition $(n=15)$. The mean age of the older sample (13 male, 13 female) was $7.65(S D=$ 0.98) years. Twelve of the older children were in the accurate gesture condition and 14 in the misleading gesture condition.

\section{Design}

All children viewed a target video and completed three stages of the study; pre-interview recall, interview, and (following a short three-minute distractor task) post-interview recall. At interview, children were allocated randomly to either an accurate gesture or misleading gesture condition (between subjects).

\section{Materials}

The Adaptive Language Inventory (ALI; Feagans \& Farrans, 1997) was used to measure each child’s language skills. The ALI consists of 18 items regarding: comprehension (ability to work with little help from the teacher), production (the ability to communicate logically), rephrasing (the ability to respond to questions in a thoughtful way), spontaneity of speech (talks to peers easily), listening skills, (listens to others), and fluency (easily understood by peers). Teachers were asked to indicate the extent to which they agreed with each statement on a 5 -point likert scale (from $1=$ well below average, to $5=$ well above average). A mean ALI score was calculated for each child, providing an indication of the child's language abilities relative to their peers. The 
Running head: Misleading gesture in children's eyewitness interviews

ALI has been used in previous studies to understand the relationship between verbal language ability and suggestibility (Clarke-Stewart et al., 2004; Kulkofsky \& Kelmfuss, 2008).

A short vignette was created for the experiment that featured a man and woman roller-skating in different directions, performing tricks and using equipment including a hula-hoop and a ball. The man fell over, was helped up by the woman and indicated that he had hurt his elbow. The actors had a positive affect and the video was designed to be appealing to children. There was no dialogue in the video, only an upbeat soundtrack from children's televisions shows. The vignette was presented on a laptop computer and was 1 minute and 28 seconds in duration. A 'flip' Ultra HD video camera was used to record the children. Children were given a picture of a house to color in and some crayons during the distractor task.

\section{Procedure}

Teachers were provided with the ALI in advance of testing and asked to complete these for the participating children. Children were tested individually in a quiet area of the nursery, children's center or school. The children were seated at a table opposite the experimenter with a laptop on the table in front of them. Children were told that they would watch a short video twice and would need to watch it carefully as they would be talking about it afterwards. The vignette was played twice and the experimenter then asked the child to tell them what happened in the video (pre-interview recall). Standard prompting questions were used if the child was unresponsive or said "I don’t know”, including "What else did they do?” and "Can you remember anything else?”

Children were then interviewed and were asked eight focused questions about the vignette. The verbal content of these questions remained identical in both conditions, however the gestures accompanying these questions varied by condition. In the accurate gesture condition, the experimenter accompanied her questions with gestures that were concordant with what happened in the video. For example, "What was the lady wearing?” plus a gesture to depict putting on a hat (both hands in fists above the head pulled downwards to the ears). In the misleading gesture condition, the interviewer produced gestures that were discordant with the events of the video and were therefore misleading. "What was the lady wearing?” plus a 'put on gloves' gesture (left hand wrapped around right hand, left hand moves down towards the right wrist whilst the right hand opens up and fingers spread, as though putting on a glove; see Appendix for a full list of questions and the 
Running head: Misleading gesture in children's eyewitness interviews

accompanying gestures). Children were given a picture to color in for three minutes as a distractor task before being asked to recall everything that they could remember from the video (post-interview recall). As per the pre-interview recall, standard prompting questions were used if necessary. Children were thanked and praised for taking part and were awarded a sticker for their participation and given the picture that they had colored in to take home.

\section{Coding children's verbal responses.}

Pre-interview recall. Each piece of information that the child recounted during their pre-interview recall was coded as either correct or incorrect. A correct response was coded if the child recalled something that occurred in the video, and an incorrect response was coded if the child recalled something that did not. The total number of correct pieces of information was then calculated for each child to provide a measure of their baseline accuracy.

Interview. Children's verbal responses to the questions during the suggestive interview were coded as correct or incorrect. In addition, children's incorrect responses were coded to identify responses that were consistent with the interviewer's misleading gesture. For example, in the misleading gesture condition the question “What was the lady wearing?” was accompanied by a 'putting on gloves' gesture. If the child gave an incorrect response of 'gloves', this was coded as consistent with the misleading gesture. The total number of questions (out of 8) that children provided incorrect answers consistent with the misleading gestured information was calculated as an index of gestural misinformation.

Post-interview recall. The number of pieces of information that the child recounted in their postinterview recall was recorded. Each piece of information was further coded to identify verbal information that did not appear in the children's pre-interview recall and was therefore new in their post-interview recall. Gesture suggested new information was coded when the child included information at post-interview recall that was not mentioned at pre-interview recall and was consistent with the information conveyed in gesture by the experimenter during the suggestive interview (therefore this could have been conveyed by misleading or accurate gestures, depending on condition). 
Running head: Misleading gesture in children's eyewitness interviews

Coding children's nonverbal responses. The videotaped sessions were coded using the Noldus Observer XT software; a computer-aided coding software for observational analysis. The gestures that children produced alongside their spoken responses were coded. For the purpose of this study, only iconic gestures were coded. Iconic gestures convey semantic information and, in accordance with McNeill (1992), were coded from the moment the child's hands deviated from equilibrium to the moment they returned to equilibrium. Each iconic gesture was further coded to indicate whether it was a replication of a gesture modelled by the experimenter. A second coder coded $10 \%$ of the sample (six participants) for gesture production. Agreement between the two coders was excellent; Kappa $=.80$ with $p<.001$.

\section{Results}

\section{Were Children Misled by Gesture?}

To assess whether children were misled by gesture during questioning, we calculated the number of questions that children answered incorrectly that were consistent with the misleading gestured information, and examined the impact of age and language ability on children's susceptibility to being misled by gesture when questioned immediately after viewing the event.

Of the younger sample, 14 of 15 children were misled by gesture. The mean number of answers (out of a maximum of 8$)$ that assented to misleading gesture was $2.60(S D=1.55$, mode $=4$, range $0-5)$. In the older sample, 11 out of 14 children were misled on at least one question, with a mean of $2.00(S D=1.52$, mode $=2$, range 0-5) answers assenting to information conveyed by misleading gesture. Both age groups appeared to be equally vulnerable, $t(27)=1.05, p=.30$. Thus, even when questioned immediately after the event, when the memory trace is presumed strong, there is evidence of a gestural misinformation effect in both younger and older children. We considered whether particular gestures were more misleading than others and calculated the number of times that children were misled by each gesture (Figure 1). While every gesture misled at least one child, the gestures that exerted greatest influence (for both older and younger children) were the 'throw' and 'eating' gestures (see Figure 1). 
Running head: Misleading gesture in children's eyewitness interviews

\section{INSERT FIGURE 1 ABOUT HERE}

We went on to consider the relationship between children's baseline accuracy and their suggestibility to nonverbal influence. Older children reported a mean of $9.38(S D=4.14)$ pieces of information in their preinterview recall, and younger children $3.70(S D=1.90)$, a significant difference, $t(33.96)=-6.44, p<.01$, Cohen's $d=1.76$. Even though the older children provided significantly more details in their first narrative than younger children, the two groups made the same number of errors and were equally misled by gesture. We then conducted within-groups analyses to test the relationship between children's baseline accuracy and the extent to which they were misled by gesture. There was no significant correlation between baseline accuracy and the number of answers consistent with misleading gestured information for the sample as a whole; $r(27)=-.06, p=$ .77 , the younger sample; $r(13)=.07, p=.79$, or the older sample; $r(12)=.19, p=.51$. Thus, even children who initially recalled a high number of correct pieces of information about the event were open to adopt additional misinformation gleaned from gesture into their narratives.

Next, we examined whether language ability buffered children against the effects of misleading gesture. The mean ALI score of the younger sample was $3.47(S D=0.65)$ and the mean score of the older group was $3.55(S D=0.70)$. No significant correlations were found between children's ALI scores and the number of answers that were consistent with misleading gestured information for the sample as a whole, $r(27)=-.07, p=$ .72 , the younger group, $r(13)=-.05, p=.86$ or the older group, $r(12)=-.01, p=.97$.

\section{Did Children Incorporate Gestured Information Into Their Verbal Narratives?}

The previous analysis revealed that children were misled into giving incorrect answers to questions accompanied by misleading gestures. The focus of this analysis was to determine whether the children's subsequent verbal narratives of the event would demonstrate that children had accommodated this (inaccurate) nonverbal information into their representations of the event. To examine this, we identified cases where the children's post-interview recall narratives included information that was absent from their pre-interview recall but consistent with the gestures of the interviewer (gesture suggested new information). The means are reported in Table 1. We found that children did indeed incorporate information gleaned from misleading gestures into their verbal narratives of the event. Six out of the 14 younger children who were misled by gesture during questioning incorporated the misleading gestured information into their post-interview recall. In the older 
Running head: Misleading gesture in children's eyewitness interviews

sample, the occurrence was less frequent, however 4 of the 14 older children reported at least one piece of gesture-misled information. We considered what type of misleading information was more likely to be incorporated into children's post-interview narratives and counted the number of times that children included misinformation from the different gestures. Children reported people in the video eating (older children $n=2$, younger children $n=4$ ), hurting their head (younger children $n=2$ ), the lady throwing a ball (older children $n=$ 1, younger children $n=1$ ), juggling (younger children $n=1$ ), wearing gloves (older children $n=1$ ), having a triangle on their clothing (older children $n=1$ ), and skating in a circular motion (older children $n=1$ ).

A two-way Analysis of Variance (ANOVA) was conducted to test the effect of condition (accurate or misleading gestures) and age (younger or older) on gesture suggested new information. A sensitivity power analysis was conducted with G*power (Faul, Erdfelder, Lang, \& Buchner 2007) which revealed that the test was sufficiently powered (80\%) to detect an effect of .38 magnitude, with alpha $=.05$. There was a significant main effect for condition, $F(1,52)=16.44, p<.01$, with children incorporating significantly more information from accurate gestures into their narratives than from misleading gestures. However, there was no main effect for age, $F(1,52)=0.48, p=.49$, and no significant interaction between age and condition $F(1,52)=1.07, p=$ .31.

\section{INSERT TABLE 1 ABOUT HERE}

Children's replication of interviewer's gestures. Children replicated the interviewer's gestures during questioning as well as during the post-interview recall stage. The total numbers of gestures produced by children during interview and post-interview are presented in Table 2, along with the number of these gestures that were replications of the interviewer's gestures. If the child had produced a gesture at pre-test then this was not counted as a replication of the interviewer's gesture if they produced the same gesture during questioning or at post-interview recall. 
There was no significant relationship between ALI score and the number of gestures replicated at interview $[r(49)=.05, p=.72]$ or at post-interview recall $[r(51)=.21, p=.13]$, thus ALI score was not included in subsequent analyses. A two-way ANOVA was conducted to assess the impact of age (younger vs. older) and condition (accurate vs. misleading gesture) on the mean number of replicated gestures produced at questioning (Table 2). There was a significant main effect of age; $F(1,47)=5.89, p=.02$, and a significant main effect of condition; $F(1,47)=16.63, p=.02$. There was no significant interaction $(p>.05)$. Thus, children replicated significantly more accurate gestures than misleading gestures, and younger children significantly more gestures than older children. The same analysis was repeated with the number of replicated gestures at post-interview recall as the dependent variable but no significant effects were found $(p>.05)$.

Finally, we examined whether replicating the interviewer’s misleading gestures during questioning would predict whether children would report more gesture-misled information in their post-interview recall. For the younger children, there was no significant relationship between the number of replicated misleading gestures they produced during questioning and the amount of gesture misled information they reported verbally at post-interview recall, $r(13)=.24, p=.40$. However, there was a significant positive relationship for the older children, $r(10)=.59, p=.04$, revealing the more children replicated misleading gestures during questioning the more incorrect information they would report subsequently in their verbal narratives.

\section{Discussion}

Our findings demonstrate the remarkable robustness of the gestural misinformation effect in child eyewitness interviews and confirm the effects reported in previous research (Broaders \& Goldin-Meadow, 2010). We have tested the strength of the gestural misinformation effect to reveal that gesture pervades to mislead under conditions that would normally buffer children against verbal suggestibility. Children were susceptible to gestural misinformation even when questioned immediately after witnessing an event, when the memory trace is presumed strong. There were no significant main effects of age; younger children were no more vulnerable to nonverbal influence than older children. However, a developmental trend might be suggested; $93 \%$ of 2-4 year old children were misled by gesture compared to 79\% of 6-9 year old children. Broaders and Goldin-Meadow reported 77\% of the 5-6 year old children in their sample subscribed to at least 
Running head: Misleading gesture in children's eyewitness interviews

one untrue suggestion made in the open-ended questions produced with gestures. This emerging trend is in-line with evidence identifying 3-4 year olds as most vulnerable to the effects of misleading verbal information (Ceci \& Bruck, 1993; Ceci, Ross \& Toglia, 1987; Goodman \& Reed, 1986; Poole \& Lindsay, 1995).

Interestingly, younger children in our sample (but not older) went beyond assenting to misleading gesture to infer additional details. When asked, “What did they have at the end?” accompanied by an eating gesture, younger children’s responses included: “A yummy sandwich”; “They eat (sic) ice cream”; “Sweets.... lollipops”; and "Apples”. In the video, the actors in fact had a drink. The older children who were misled on this question would typically just say that they were eating, without elaborating on what they were eating.

In general, children's susceptibility to gestural misinformation was high. While previous research has demonstrated that children's verbal language ability (specifically, high ALI scores) protects them against the misleading effects of verbal influence (e.g., Clarke-Stewart et al., 2004; Kulfosky \& Klemfuss, 2008), we found no evidence that verbal language ability protects children against the misleading effects of nonverbal influence. This is the first study to probe the gestural misinformation effect by considering the contribution of factors known to protect against verbal suggestibility. While the findings from this exploratory study are novel and have potential for serious implications, caution must be applied in interpretation due to the relatively small sample size. Nonetheless, our study raises significant questions for future research that warrant investigation in larger samples.

The misleading information that was conveyed by the interviewer's gestures appeared in children’s subsequent verbal narratives of the event, much like it does for adults (Kelly, Barr, Church \& Lynch, 1999). Furthermore, children replicated the interviewer's gestures and doing so predicted the extent to which this information would appear subsequently in the older children's verbal narratives of the event (but not the younger children). Why are children highly susceptible to nonverbal influence? While adults glean information from gesture (either wittingly or not, though typically not- e.g., Kelly et al., 1999), the gesture channel is an important source of information for children. Given that children's verbal abilities are less proficient than adults, gesture offers children a more accessible route to communication (Hostetter, 2011). Furthermore, younger children's working memories are more reliant on the visuospatial sketchpad, switching to verbal rehearsal strategies around the age of seven (Hitch, Halliday, Schaafstal, \& Schraagen, 1988). While speech is 
Running head: Misleading gesture in children's eyewitness interviews

bound by “a linear-segmented hierarchical linguistic structure, gestures provide 'a global-synthetic' representation” (McNeill, 1992, p.245) and have been claimed to "tap visual and/or proprioceptive sensory memories of an object experience” (Capone, 2007, p.741), providing a channel compatible with children's working memory limitations.

Indeed, a recent meta-analysis by Hostetter (2011) examined the effect sizes from 63 studies in which listeners' understanding of a message was compared when speech was presented alone, with speech accompanied by gesture. Significantly larger effects of gesture were found in studies that included children as listeners than studies of adults, thus children are claimed to reap greater benefit from multi-modal communication and are highly sensitive to information conveyed in the gesture channel. Seeing a speaker's gestures is beneficial for children as gestures convey information supplementary or complementary to that conveyed by speech, as demonstrated by a wealth of research examining instructors' gestures in the classroom (Alibali \& Nathan, 2007; Church, Ayman-Nolley, \& Mahootian, 2004; Goldin-Meadow, Kim, \& Singer, 1999; Singer \& Goldin-Meadow, 2005; Valenzeno, Alibali, Klatzky, 2003). Indeed, we found that accompanying questions with gestures that conveyed accurate information facilitated children's verbal recall. However, this sensitivity to the gestural channel is not always beneficial and can put children at a disadvantage when information conveyed in gesture is unreliable, as first identified by Broaders and Goldin-Meadow (2010).

Our findings indicate that children have a limited capacity to ignore information conveyed by gesture that contradicts their event memory. While the children in our study were more likely to pick-up accurate information conveyed by the interviewer's gestures, nearly all of the children still reported at least one piece of false information gleaned from the interviewer's misleading gestures. While this would suggest children are able to mediate misinformation effects to some degree, we found little evidence of this through further exploration of the data. In summary, children were not attending to and assimilating the information conveyed by the interviewer's gestures indiscriminately, yet were clearly limited in their ability to reject misleading information. This could be explained partly by the perceived authority of an adult interviewer (Smith \& Ellsworth, 1987). However, given the difference in the amount of information extracted from accurate and misleading gestures, the data suggest that children would not have explicitly perceived the interviewer's gestures as an ostensive prompt to guide them to provide the 'correct' answer. 
Running head: Misleading gesture in children's eyewitness interviews

The gestural misinformation effect is robust and warrants further investigation to better understand the impact of this effect. We found that the rate at which older children replicated the interviewer's gestures during questioning predicted the extent to which they would report this information verbally when asked to recount what they remembered later on. However, the same relation did not hold for the younger children. Thus, children are prone to replicating misleading gestures yet may not verbalize this inaccurate information. We are interested to know whether adults are sensitive to this (mis)information conveyed in children's gestures. We would expect them to be sensitive to the information that children convey uniquely in their gesture as studies have found that adults attribute understanding to a child when that information is expressed only in the child's gestures (Alibali, Flevares \& Goldin-Meadow, 1997; Goldin-Meadow, Wein \& Chang, 1992). If we ask adults to watch a child recount their memory of an event, then ask the adults to tell us what the child witnessed, will they include (false) information that children conveyed in gesture only? This has important implications for the legal context and is a question we'll be addressing in our ongoing research.

We have presented findings to demonstrate that the gestural misinformation effect is resilient to factors that are known to diminish the influence of verbal suggestibility (memory trace strength, age, and verbal ability). We found that children copied the interviewer's gestures during questioning and doing so predicted the extent to which that erroneous information would appear in older children's subsequent verbal narratives.

Evidence suggests that by encoding information in dual modalities, gesturing produces stronger and more robust memory traces (Cook, Mitchell, \& Goldin-Meadow, 2008; Glenberg, Gutierrez, Levin, Japuntich, \& Kaschak, 2004; Stevanoni \& Salmon, 2005). Children understand stories better if they physically enact them (Glenberg et al., 2004) and children’s learning of new concepts has been demonstrated to be longer lasting if encouraged to gesture at the point of instruction (Cook, Mitchell, \& Goldin-Meadow, 2008). Encouraging children to gesture when remembering has been shown to enhance event recall (Stevanoni \& Salmon, 2005). Thus, encoding information in a nonverbal format may strengthen the encoding of a false memory. In this way, the gestural misinformation effect may be more pervasive than verbal influence. A recent study has compared the effects of misleading gesture against those of speech in adult witnesses directly, concluding that gestures are just as likely as speech to prompt participants into reporting inaccurate, misleading details (Gurney, in press). It remains to be tested whether the same holds for children. 
Running head: Misleading gesture in children's eyewitness interviews

The robustness of the gestural misinformation effect has serious implications for child eyewitnesses. Before an official forensically interviews a child, an adult, perhaps a teacher or a parent, would likely have questioned that child soon after the witnessed event. The adult may have assumptions about the details of the event that the child has witnessed which they may convey (unwittingly or not) via their gestures. For example, the adult may assume that a perpetrator was wearing a hoodie, for instance, and convey this information unknowingly with their hand gestures. Our study has shown that the gestures that the child is exposed to at this point can seriously infiltrate the child's memory of the event and the (mis)information conveyed in the adult's gestures is likely to appear in the child's verbal account in the forensic interview. Gesture misleads children under conditions that would normally protect children from verbal influence. The gestural misinformation effect is robust, resilient and has greater potential to influence than suggestive speech. 
Running head: Misleading gesture in children's eyewitness interviews

References

Alexander, K. W., Goodman, G. S., Schaaf, J. M., Edelstein, R. S., Quas, J. A., \& Shaver, P. R. (2002). The role of attachment and cognitive inhibition in children's memory and suggestibility for a stressful event. Journal of Experimental Child Psychology, 83, 262-290.

Alibali, M. W., Flevares, L. M., \& Goldin-Meadow, S. (1997). Assessing knowledge conveyed in gesture: Do teachers have the upper hand? Journal of Educational Psychology, 89(1), 183.

Alibali, M. W., \& Nathan, M. J. (2007). Teachers’ gestures as a means of scaffolding students' understanding: Evidence from an early algebra lesson. Video Research in the Learning Sciences, 349-365.

Broaders, S. C., \& Goldin-Meadow, S. (2010). Truth is at hand: How gesture adds information during investigative interviews. Psychological Science, 21, 623-628.

Bruck, M., \& Melnyk, L. (2004). Individual differences in children’s suggestibility: A review and synthesis. Applied Cognitive Psychology, 18, 947-996.

Burgwyn-Bailes, E., Baker-Ward, L., Gordon, B. N., \& Ornstein, P. A. (2001). Children’s memory for emergency medical treatment after one year: The impact of individual difference variables on recall and suggestibility. Applied Cognitive Psychology. Special Issue: Trauma, Stress, and Autobiographical Memory, 15(7), S25-S48. DOI: 10.1002/Acp.833.

Capone, N. C. (2007). Tapping toddlers' evolving semantic representation via gesture. Journal Of Speech Language snd Hearing Research, 50(3), 732-745.

Ceci, S.J., \& Bruck, M. (1993). Suggestibility of the child witness. A historical review and synthesis. Psychological Bulletin, 113, 403-439.

Ceci, S.J., \& Bruck, M. (1999). The suggestibility of children’s memory. Annual Review of Psychology. 50, 419-39.

Ceci, S. J., Ross, D. F., \& Toglia, M. P. (1987). Suggestibility of children’s memory: Psycholegal implications. Journal of Experimental Psychology: General, 116, 38-49. 
Running head: Misleading gesture in children's eyewitness interviews

Church, R. B., Ayman-Nolley, S., \& Mahootian, S. (2004). The role of gesture in bilingual education: Does gesture enhance learning?. International Journal of Bilingual Education and Bilingualism, 7(4), 303-319.

Clarke-Stewart, K. A., Malloy, L. C., \& Allhusen, V. D. (2004). Verbal ability, self-control, and close relationships with parents protect children against misleading suggestions. Applied Cognitive Psychology, 18, 1037-1058.

Cook, S., Mitchell, Z., \& Goldin-Meadow, S. (2008). Gesturing makes learning last. Cognition, 106, 10471058.

Faul, F., Erdfelder, E., Lang, A.-G., \& Buchner, A. (2007). G*Power 3: A flexible statistical power analysis program for the social, behavioral, and biomedical sciences. Behavior Research Methods, 39, 175-191

Feagans, L. V., \& Farrans, D. C. (1997). Adaptive Language Inventory (ALI), Chapel Hill, NC: Frank Porter Graham Child Development Center.

Geddie, L., Fradin, S., \& Beer, J. (2000). Child Characteristics Which Impact Accuracy Of Recall And Suggestibility In Preschoolers: Is Age The Best Predictor? Child Abuse \& Neglect, 24, 223-235.

Glenberg, A. M., Gutierrez, T., Levin, J. R., Japuntich, S., \& Kaschak, M. P. (2004). Activity and imagined activity can enhance young childrens’ reading comprehension. Journal of Educational Psychology, 96, 424-436.

Goldin-Meadow, S., Kim, S., \& Singer, M. (1999). What the teacher's hands tell the student's mind about math. Journal of Educational Psychology, 91, 720-730.

Goldin-Meadow, S., Wein, D., \& Chang, C. (1992). Assessing knowledge through gesture: Using children's hands to read their minds. Cognition and Instruction, 9(3), 201-219. 
Running head: Misleading gesture in children's eyewitness interviews

Goodman, G. S., \& Reed, R. S. (1986). Age differences in eyewitness testimony. Law and Human Behavior, 10, 317-332.

Gurney, D. J. (in press). What's left unsaid: How nonverbal influence compares with verbal influence.

Psychiatry, Psychology and Law. DOI: 10.1080/13218719.2014.985624

Gurney, D. J., Pine, K. J., \& Wiseman, R. (2013). The gestural misinformation effect: Skewing eyewitness testimony through gesture. American Journal of Psychology, 126(3), 301-314. DOI: 10.5406/Amerjpsyc.126.3.0301

Gullberg, M., \& Holmqvist, K. (2006). What speakers do And what addressees look at: Visual attention to gestures in human interaction live and on video. Pragmatics \& Cognition, 14(1), 53-82.

Gullberg, M., \& Kita, S. (2009). Attention to speech-accompanying gestures: Eye movements and information uptake. Journal of Nonverbal Behavior, 1-27.

Hitch, G. J., Halliday, S., Schaafstal, A. M., \& Schraagen, J. M. C. (1988). Visual working memory in young children. Memory \& Cognition, 16, 120-132.

Hostetter, A. B. (2011). When do gestures communicate? A meta-analysis. Psychological Bulletin, 137, 297315.

Kassin, S. M., Ellsworth, P. C., \& Smith, V. L. (1989). The "general acceptance” of psychological research on eyewitness testimony: A survey of the experts. American Psychologist, 44, 1089-1098.

Kelly, S. D., Barr, D. J., Church, R. B., \& Lynch, K. (1999). Offering a hand to pragmatic understanding: The role of speech and gesture in comprehension and memory. Journal of Memory and Language, 40(4), 577-592.

King, M. A., \& Yuille, J. C. (1987). Suggestibility and the child witness. In S. J. Ceci, M. Toglia, \& D. Ross (Eds.), Children’s eyewitness memory (pp. 24-35). New York: Springer-Verlag.

Kirk, E., Pine, K. J., \& Ryder, N. (2011). I hear what you say but I see what you mean: The role of gestures in children’s pragmatic comprehension. Language and Cognitive Processes, 26, 149-170. 
Running head: Misleading gesture in children's eyewitness interviews

Klemfuss, J.Z. \& Ceci, S.J. (2012). Legal And Psychological Perspectives On Children’s Competence To Testify In Court. Developmental Review, 32, 268-286.

Kulkofsky, S., \& Klemfuss, J. Z. (2008). What the stories children tell can tell about their memory: Narrative skill and young children’s suggestibility. Developmental Psychology, 44, 1442-1456.

Loftus, E. F. (2005) Planting misinformation in the human mind: A 30-year investigation of the malleability of memory. Learning and Memory, 12, 361-365.

Loftus, E. F., \& Hoffman, H. G. (1989). Misinformation and memory: The creation of new memories. Journal of Experimental Psychology, 118, 100-104.

Marche, T. A. (1999). Memory strength affects reporting of misinformation. Journal of Experimental Child Psychology, 73, 45-71.

Marche, T. A., \& Howe, M. L. (1995). Preschoolers report misinformation despite accurate memory. Developmental Psychology, 31, 554-567.

McFarlane, F., Powell, M. B., \& Dudgeon, P. (2002). An examination of the degree to which IQ, memory performance, socio-economic status and gender predict young children’s suggestibility. Legal \& Criminological Psychology, 7, 227-239.

McNeill, D. (1992). Hand and mind: What gestures reveal about thought. Chicago: University Of Chicago Press.

Penrod, S. D., Loftus, E. F., \& Winkler, J. (1982). The reliability of eyewitness testimony: A psychological perspective. In N. Kerr \& R. Bray (Eds.), The psychology of the courtroom (pp. 119-168). New York: Academic Press.

Pezdek, K., \& Roe, C. (1995). The effect of memory trace strength on suggestibility. Journal of Experimental Child Psychology, 60, 116-128.

Poole, D. A., \& Lindsay, D. S. (1995). Interviewing preschoolers: Effects of nonsuggestive techniques, parental coding, and leading questions on reports of nonexperienced events. Journal of Experimental Child Psychology, 60, 129-154. 
Running head: Misleading gesture in children's eyewitness interviews

Roberts, K.P., \& Blades, M.(1999). Children’s memory and source monitoring of real-life and televised Events. Journal of Applied Developmental Psychology, 20, 575-596

Singer, M. A., \& Goldin-Meadow, S. (2005). Children learn when their teacher’s gestures and speech differ. Psychological Science, 16, 85-89.

Skagerberg, E. M., \& Wright, D. B. (2009). Susceptibility to postidentification feedback is affected by source credibility. Applied Cognitive Psychology, 23(4), 506-523.

Smith, V., \& Ellsworth, P. (1987). The social psychology of eyewitness accuracy: Misleading questions and communicator expertise. Journal of Applied Psychology, 72(2), 294-300.

Stevanoni, E., \& Salmon, K. (2005). Giving memory a hand: Instructing children to gesture enhances their event recall. Journal of Nonverbal Behavior, 29, 217-233.

Valenzeno, L., Alibali, M. W., \& Klatzky, R. (2003) Teachers’ gestures facilitate students’ learning: A lesson in symmetry. Contemporary Educational Psychology, 28, 187-204.

Warren, A. R., Hulse-Trotter, K. \& Tubbs, E. (1991) Inducing resistance to suggestibility in children. Law and Human Behavior, 15, 273-285.

Young, K., Powell, M. B., \& Dudgeon, P. (2003). Individual differences in children’s suggestibility: A comparison between intellectually disabled and mainstream samples. Personality \& Individual Differences, 35, 31-49. 
Running head: Misleading gesture in children's eyewitness interviews

Table 1.

Mean (SD) Gesture suggested new information reported at post-interview recall ${ }^{1}$

\begin{tabular}{llll}
\hline Sample & Condition & $n$ & Mean (SD) \\
\hline Younger & Accurate & 15 & $1.47(1.55)$ \\
& Misleading & 15 & $0.93(1.03)$ \\
Older & Accurate & 12 & $2.00(1.28)$ \\
& Misleading & 14 & $0.50(1.09)$ \\
\hline
\end{tabular}

${ }^{1}$ information reported at post-interview recall that was (a) not reported at pre-interview recall and (b) consistent with the information conveyed in gesture by the interviewer. 
Running head: Misleading gesture in children's eyewitness interviews

Table 2.

Mean (SD) gesture production by sample, condition, and experimental stage.

\begin{tabular}{|c|c|c|c|c|c|c|}
\hline \multirow[t]{2}{*}{ Sample } & \multirow[t]{2}{*}{ Condition } & \multirow[t]{2}{*}{$n$} & \multicolumn{2}{|c|}{ Interview } & \multicolumn{2}{|c|}{ Post Interview } \\
\hline & & & $\begin{array}{l}\text { Total no. } \\
\text { gestures }\end{array}$ & $\begin{array}{l}\text { Total no. } \\
\text { gestures } \\
\text { replicated } \\
\text { from } \\
\text { interviewer }\end{array}$ & $\begin{array}{l}\text { Total no. } \\
\text { gestures }\end{array}$ & $\begin{array}{c}\text { Total no. } \\
\text { gestures } \\
\text { replicated from } \\
\text { interviewer }\end{array}$ \\
\hline \multirow[t]{2}{*}{ Younger } & Accurate & 15 & $4.21(3.14)$ & $2.93(2.30)$ & $0.93(1.38)$ & $0.64(1.23)$ \\
\hline & Misleading & 15 & $2.47(2.88)$ & $1.13(1.60)$ & $0.40(0.63)$ & $0.13(0.35)$ \\
\hline \multirow[t]{2}{*}{ Older } & Accurate & 12 & $2.10(3.60)$ & $1.10(1.20)$ & $1.00(1.25)$ & $0.40(0.52)$ \\
\hline & Misleading & 14 & 2.58 (2.39) & $0.58(1.44)$ & $2.25(1.71)$ & $0.25(0.62)$ \\
\hline
\end{tabular}


Running head: Misleading gesture in children's eyewitness interviews

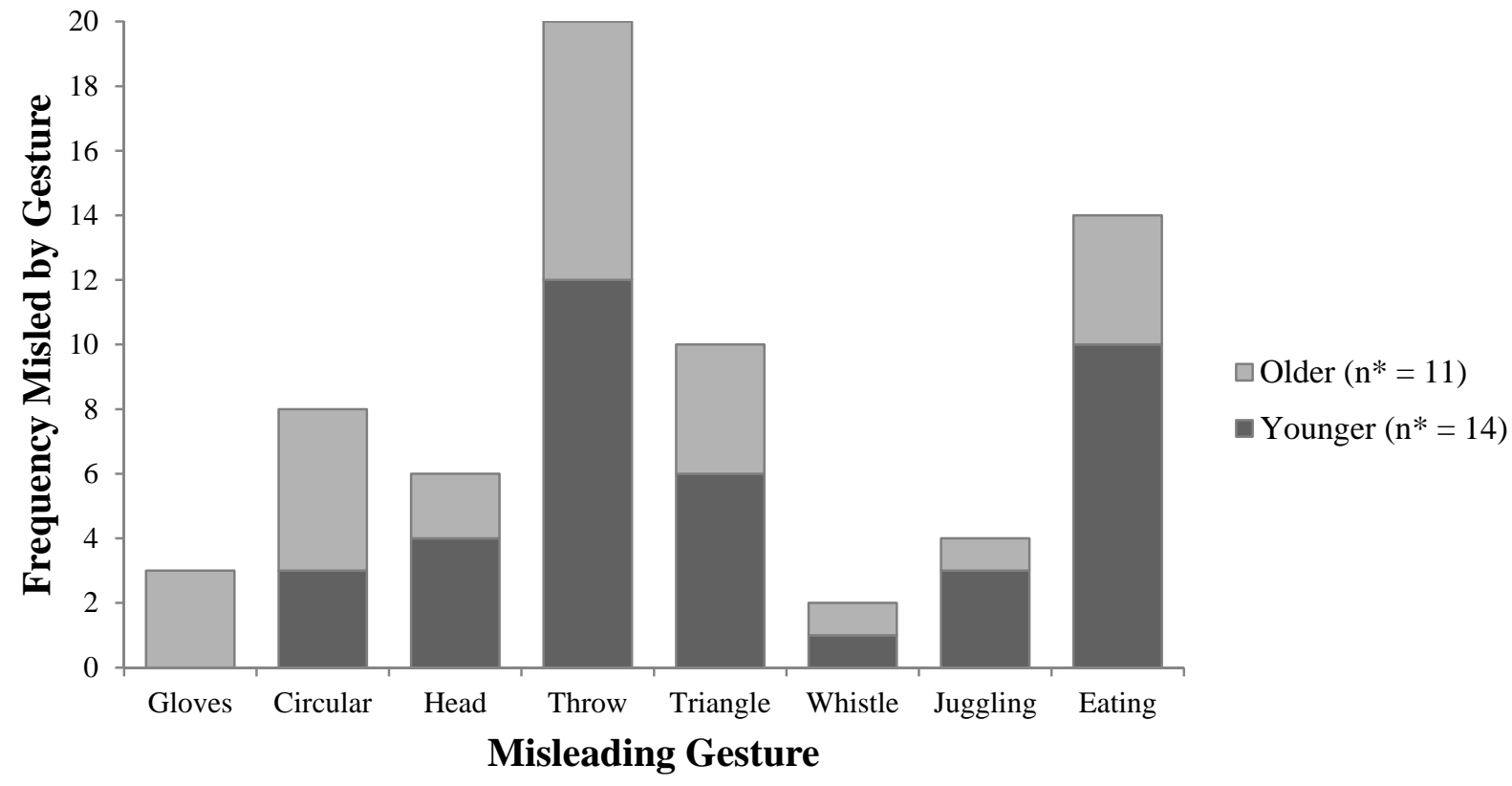

Figure 1. The number of times older and younger children were misled by individual gestures during questioning

*= number of children misled by at least one gesture during questioning 
Running head: Misleading gesture in children's eyewitness interviews

\section{Appendix}

Appendix I: Suggestive Interview Questions and Accompanying Gestures

1. What was the lady wearing?

Accurate Gesture Condition: put on hat gesture

Misleading Gesture Condition: put on gloves gesture

2. Which way did they skate?

Accurate Gesture Condition: backwards and forwards gesture

Misleading Gesture Condition: circular gesture

3. Where did the man hurt himself?

Accurate Gesture Condition: elbow tap

Misleading Gesture Condition: head tap

4. What did the lady do with the ball?

Accurate Gesture Condition: catch gesture

Misleading Gesture Condition: throw gesture

5. What shape was on the lady's top?

Accurate Gesture Condition: circle gesture

Misleading Gesture Condition: triangle gesture

6. What else did they do?

Accurate Gesture Condition: Hula Hoop gesture 
Running head: Misleading gesture in children's eyewitness interviews

Misleading Gesture Condition: blow whistle gesture

7.Then what did the man do?

Accurate Gesture Condition: mobile phone gesture

Misleading Gesture Condition: juggle gesture

8. What did they have at the end?

Accurate Gesture Condition: drinking gesture

Misleading Gesture Condition: eating gesture 